\title{
Comparative study of the photogrammetry process in different hardware
}

XXIV International Conference

Simone Helena Tanoue Vizioli

Instituto de Arquitetura e Urbanismo da Universidade de São Paulo | Brasil | simonehtv@usp.br

\author{
Andrea Adami \\ Politécnico di Milano | Itália | andrea.adami@polimi.it \\ Laura Hiilesmaa \\ Instituto de Arquitetura e Urbanismo da Universidade de São Paulo | Brasil | \\ laura.hiilesmaa@usp.br
}

Leonardo Chieppe Carvalho

Instituto de Ciências Matemáticas e de Computação da Universidade de São Paulo | Brasil | leonardo.chieppe.carvalho@usp.br

\begin{abstract}
Photogrammetry is not a new technology, but its use, nowadays is being potentiated either in urban projects, architecture, restoration of historical heritage and archaeological documentation, among others. In this research, the use of photogrammetry as an important tool in the preservation and documentation of cultural heritage will be addressed. The specific objective of the article is to analyze the photogrammetry modeling process: processing time, resolution quality and generated products, in 2 different hardware; highlighting its potentialities and deficiencies. The object of study is the Casa do Pinhal, an important historical heritage for the identity and memory of the city of São Carlos (SP-Brazil).
\end{abstract}

Keywords: Photogrammetry; 3D Modeling; Metashape; Heritage education; Point cloud.

\section{INTRODUÇÃO}

Na evolução histórica e tecnológica, é preciso definir não mais o papel da máquina, mas sim, o papel do homem. 0 homem já atravessou grandes revoluções e vive a Revolução Digital, que vem proporcionando mudanças na sociedade e em seu modo de vida. A Revolução Digital (Righi, 2008) incluiu os computadores e a internet nas atividades mais básicas dos seres humanos proporcionando uma mudança nos modos de produção e de desenvolvimento de produtos, ampliando as fronteiras de conhecimento e aumentando exponencialmente a capacidade de processamento e de resolução de tarefas complexas.

Qual o atual momento? Segundo Carpo (2017), nos anos 1990, pode-se dizer que as novas máquinas digitais eram usadas para implementar a velha ciência que se conhecia, toda a ciência era transferida para as novas plataformas computacionais que estavam sendo descobertas. Hoje, pelo contrário, os computadores podem funcionar melhor e mais rapidamente se, a eles, for permitido seguir um método diferente, não humano e pós-científico. Carpo afirma ainda que, em um sentido metafórico, os computadores estão agora desenvolvendo sua própria ciência - um novo tipo de ciência. Nos anos 1990 havia uma certa falta de dados computacionais e hoje, lida-se com um excesso de dados. Há ainda uma outra relação, aquela na qual o homem não mais controla os dados do computador, a simbiose homem-computador é dominada pela máquina, e se o homem assim o permitir, a máquina caminhará sozinha para a sua própria concepção. Não se pretende aqui discorrer sobre a tomada do mundo pelas máquinas, mas sim, sobre se ter o controle das inúmeras operações não humanas que o computador realiza, ter consciência de seu uso, para quê, quando e por que fazêlo.

Este artigo é uma continuidade dos resultados da oficina interdisciplinar "Fotogrametria + Patrimônio" (Almeida; Silva; Vizioli, 2019), realizada entre os dias 24 e 27 de abril de 2019. Esta atividade teve como objetivo o estudo e a experimentação da técnica de fotogrametria - conhecida também como mapeamento digital - visando obter um modelo tridimensional que pudesse ser usado para a documentação e educação patrimonial de um patrimônio cultural arquitetônico. Foi adotado como objeto de estudo a Casa do Pinhal, localizada na cidade de São Carlos (SP), pertencente a um conjunto arquitetônico tombado desde 1987 pelo Instituto do Patrimônio Histórico e Artístico Nacional (IPHAN). O workshop contou com a participação de 18 alunos de graduação, 8 alunos de pós-graduação em Arquitetura e Urbanismo do IAU USP e contou com a presença de Andrea Adami, Professor convidado do Politécnico di Milano que pôde instruir os estudantes por meio de palestra e exercícios práticos sobre mapeamentos digitais. 
A partir do final do século $X X$, os rápidos avanços nas tecnologias de digitalização tridimensional por nuvens de pontos se mostraram grandes aliados do processo de documentação patrimonial, com o desenvolvimento de tecnologias como laser scanner e fotogrametria. Ambas têm se mostrado revolucionárias, ao reproduzir o objeto de maneira extremamente precisa através de um modelo digital baseado em dados levantados in loco. Essa representação, ao contrário de softwares que dependem de medidas empíricas, pode revelar tridimensionalmente e com uma precisão milimétrica irregularidades, patologias e camadas de história. Seja pela facilidade de aquisição e geração de dados, pela extrema precisão ou pela crescente disponibilidade, ambas têm se mostrado o futuro das técnicas de digitalização tridimensional de patrimônios. Por meio de processos automatizados e digitais, conseguem recriar objetos das mais diferentes escalas em modelos tridimensionais digitais, e estão se tornando cada vez mais acessíveis. "A tecnologia, neste ponto, está ultrapassando a metodologia, desafiando as práticas acadêmicas e profissionais a se manterem atualizadas" (Warden, 2009, p.8, tradução do autor).

Nos últimos anos foi observada uma crescente importância dada ao patrimônio arquitetônico. Para tanto, "A documentação representa uma parte importante e essencial no processo de preservação do patrimônio e da memória, uma vez que qualquer atividade a ser desenvolvida, seja na conservação ou na restauração de sítios e monumentos históricos, requer seu registro o mais completo e preciso possível." (Amorim; Groetelaars, 2008, p. 93). Porém, o tema não tem sido muito discutido nos Congressos Sigradi: de 2003 até 2008 a palavra-chave "Heritage" comparece apenas em quatorze artigos e a palavra-chave "Photogrammetry" resulta em apenas dois artigos, 2016 e 2018 (Sperling et al, 2019).

A importância da documentação digital é reforçada em casos extremos como o do Templo de Bel, em Palmira na Síria, destruído em 2015 (Wahbeh, Nebiker, Fangi, 2016), em um ato terrorista ou da Catedral de Notre Dame que, em 2019, teve a maior parte de seu telhado destruído por um incêndio. Antes dos danos sofridos, ambos monumentos haviam sido mapeados por tecnologias de levantamento digital. O templo por meio da fotogrametria e a catedral por meio do laser scanner. Peterson (2019) explica que em uma estrutura como a Notre-Dame, construída ao longo de séculos, é quase certo que qualquer desenho ou material sobre sua construção esteja incompleto ou incorreto.

\section{OBJETIVO}

Este trabalho tem como objetivo analisar o processo de modelagem da fotogrametria: tempo de processamento, qualidade de resolução e produtos gerados, em dois diferentes hardwares; destacando suas potencialidades e deficiências. O objeto de estudo é a Casa do Pinhal, patrimônio histórico importante para a identidade e memória da cidade de São Carlos (SP-Brasil). Pretendese auxiliar os usuários do software Agisoft Metashape a ter um panorama básico de possíveis resultados, tempo de processamento e especificidades do hardware para a escolha dos parâmetros que se deseja utilizar para um determinado objetivo específico.

\section{METODOLOGIA}

Foram utilizadas 448 (quatrocentas e quarenta e oito) fotos no processamento de fotogrametria. Existem diversos softwares que podem ser usados no processo de modelagem 3D a partir de mapeamento digital, entre eles, - Agisoft Metashape, o ReCap, Scann3D e o Sphere (Bastian, 2016). Neste experimento foi utilizado o Agisoft Metashape 1.6.1.10009. Para o objetivo principal deste artigo, foram usados 2 diferentes hardwares: um notebook Intel Core i5-7300HQ CPU @ 2.5GHz; NVIDIA GeForce GTX 1050; 32GB de RAM e Samsung SSD 970 EVO (custo aproximado $\mathrm{R} \$ 5.000,00$ ) e uma workstation Intel Core i7 9700K CPU @ 3.60GHz; NVidia GeForce RTX 2070 e 64GB de RAM (custo aproximado R $\$ 15.000,00$ ). Foram registrados dados experimentais quanto à qualidade da resolução (mínima, baixa, média, alta e máxima) por etapa do workflow, o tempo de processamento em cada hardware e os produtos gerados.

\section{FOTOGRAMETRIA}

A geração do modelo digital fotogramétrico é baseada na técnica de medição tridimensional que usa fotografia para obter a localização de pontos comuns entre múltiplas fotos, de maneira que ocorra a reconstrução tridimensional de um modelo digital de um objeto.

Fassi et al. (2013) advertem que a fotogrametria é uma ferramenta de medição caracterizada por uma rápida fase de aquisição de dados, porém seguida por uma medição manual demandadora de tempo e um estágio de processamento de dados computacionais. A fase de levantamento fotográfico deve ser feita cautelosamente, já que deve haver uma cobertura de todas as superfícies do objeto, duas fotos cobrindo a mesma área devem se sobrepor em pelo menos $60 \%$ da área. As câmeras mais recomendadas para o procedimento são as DSLR de alta resolução.

No contexto de aplicações digitais, a fotogrametria possui diversos usos: a nuvem de pontos pode ser usada para auxiliar o redesenho numa aplicação BIM ou mesmo como base de modelagem CAD. O modelo (mesh) gerado pode ser importado em CAD para simplificar as passagens de modelagem. Tanto a nuvem de pontos quanto o mesh podem ser usados na documentação de patrimônios. Para impressão 3D de maquetes pode-se utilizar o mesh e, por fim, o modelo texturizado pode ser usado para renderização, seja numa maquete virtual ou simulação.

Segundo Schults (2019) vários são os usos da fotogrametria: em projetos urbanos, arquitetônicos, restauração de patrimônio histórico e documentação arqueológica, entre outros. Atualmente tem-se utilizado a fotogrametria em modelos BIM, porém, nesse processo, o modelo gerado pela fotogrametria deve ser tratado em CAD, isto é, os meshes resultados de programas como Metashape, PhotoScan, entre outros, necessitam de algum input humano para serem compatibilizados com o uso em BIM. Na Figura 1, demonstram-se possíveis caminhos do processo.

No Metashape a extração dos resultados é feita exportando os arquivos de acordo com o tipo desejado. No caso da nuvem de pontos o programa permite exportar nos formatos .ply, .las, .laz e .e57, todos compatíveis com 3D Studio Max (CAD adotado para essa pesquisa). Para a 


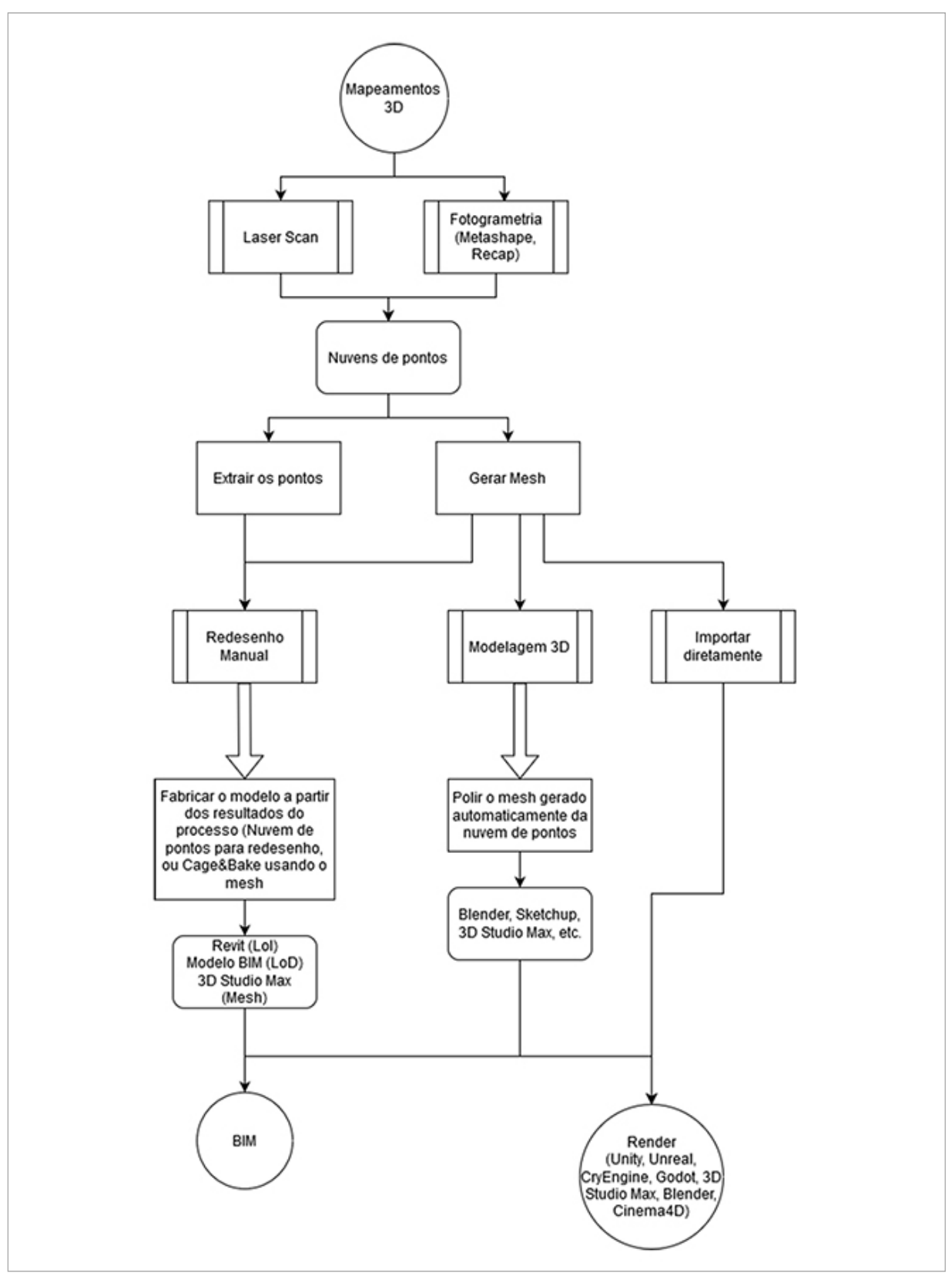

Figura 1: Workflow de mapeamento digital 3D. Fonte: autores, 2020.

extração dos modelos (mesh) o Metashape permite uma variedade de extensões distintas: .obj, .3ds, dae, .stl (para impressão 3D) e .fbx (formato ideal para uso em modelagem e renderização, dentre algumas outras opções menos utilizadas.

Diferentemente do workshop, onde cada grupo executou uma determinada fachada da Casa do Pinhal, neste trabalho, com o propósito de destacar as potencialidades e dificuldades do processo, de modo a garantir resultados objetivos comparáveis foi adotado o seguinte procedimento: o mesmo conjunto de 448 imagens foram adicionadas no Agisoft Metashape em um novo projeto como câmeras separadas. Adicionadas as imagens no projeto, seguiu-se a aba workflow do Agisoft Metashape variando-se apenas a qualidade dos processos. 
O primeiro passo correspondeu ao alinhamento das câmeras (imagens) e a formação da nuvem esparsa (sparse cloud).

O segundo passo foi a construção da dense cloud. Normalmente, seriam feitos ajustes e otimizações manuais antes desse processo, mas para evitar discrepâncias e reduzir fatores de erro humano essas etapas foram descartadas.

Após gerar a dense cloud, foi feito o processamento do mesh ou modelo, também desconsiderando qualquer etapa de ajuste ou filtragem manual de pontos.

Frequentemente, as nuvens de pontos geradas no Agisoft Metashape apresentam conjuntos de pontos indesejáveis ao modelo que se pretende gerar. Esses pontos identificados pelo programa podem ser, por exemplo, informações do ambiente onde o objeto de estudo foi fotografado. O ideal é que esses "ruídos" sejam minimizados no resultado final desejado por meio de sua seleção e remoção manual das nuvens geradas. Destacase que esse procedimento pode ser feito na sparse cloud, na dense cloud ou em ambos. Dependendo de como é feita a remoção desses pontos, haverá resultados diferentes no modelo final. Neste experimento, como dito anteriormente, essa etapa manual não foi executada para padronizar os resultados.
Na etapa final da criação do modelo completo foram feitas as texturas. Como no procedimento de texturas não existe a mesma definição de qualidade das etapas anteriores foi padronizado um mapa de $8.192 \times 8.192$ pixels para os arquivos de textura, independente da qualidade do processo.

\section{RESULTADOS E DISCUSSÃO}

Foram elaboradas Tabelas 1 e 2 das etapas de processamento de dados pelo programa Agisoft Metashape, cruzando-se dados sobre a qualidade do resultado por tempo de execução. Na primeira tabela são apresentados os dados processados no notebook e a segunda apresenta os dados na workstation. As tabelas comparativas apresentam os tempos de cada etapa do workflow, o número de pontos gerados na dense cloud, o número de faces gerados no mesh e a qualidade da textura.

Nas Tabelas 1 e 2, na linha de qualidade mínima o "não realizado" significa que a qualidade no alinhamento já estava muito baixa para gerar o modelo. No caso das qualidades mais altas, como não foi possível concluir a etapa anterior por déficit de hardware, o processo não foi realizado.

Tabela 1: Comparação entre as potencialidades do programa Agisoft Metashape no notebook.

\begin{tabular}{|c|c|c|c|c|}
\hline $\begin{array}{l}\text { Qualidade/ } \\
\text { etapa de } \\
\text { processo }\end{array}$ & $\begin{array}{l}\text { Alinhamento (sparse } \\
\text { cloud) }\end{array}$ & Dense Cloud & Mesh & Textura \\
\hline Mínima & $\begin{array}{l}1 \text { min } 44 \mathrm{~s} \\
\text { Câmeras não alinharam }\end{array}$ & Não realizado & Não realizado & $\begin{array}{l}\text { Não } \\
\text { realizado }\end{array}$ \\
\hline Baixa & $\begin{array}{l}6 \text { min } 40 \mathrm{~s} \\
\text { Alinhamento parcial. } \\
\text { Pontos estão espalhados, } \\
\text { porém formou o desenho } \\
\text { do modelo. } \\
82.879 \text { pontos }\end{array}$ & $\begin{array}{l}48 \text { min } 10 \mathrm{~s} \\
\text { Nuvem rugosa e cheia de } \\
\text { imprecisões. } \\
7.450 .745 \text { pontos }\end{array}$ & $\begin{array}{l}5 \text { min } 51 \mathrm{~s} \\
\text { Modelo precário, porém } \\
\text { razoavelmente } \\
\text { definido. Extra rugoso. } \\
165.571 \text { faces } \\
86.158 \text { vértices }\end{array}$ & $\begin{array}{l}10 \text { min } 44 \mathrm{~s} \\
\text { Texturas de } \\
\text { alta } \\
\text { qualidade. }\end{array}$ \\
\hline Média & $\begin{array}{l}14 \text { min } \\
\text { Alinhamento parcial com } \\
\text { nuvem mais bem definida. } \\
294.310 \text { pontos }\end{array}$ & $\begin{array}{l}3 \mathrm{~h} 56 \text { min } 20 \mathrm{~s} \\
\text { Nuvem mais bem definida, } \\
\text { porém com algumas } \\
\text { irregularidades. } \\
29.418 .161 \text { pontos }\end{array}$ & $\begin{array}{l}22 \text { min } 53 \mathrm{~s} \\
\text { Modelo bem definido. } \\
\text { Algumas partes } \\
\text { rugosas. } \\
1.961 .905 \text { faces } \\
994.504 \text { vértices }\end{array}$ & $\begin{array}{l}24 \text { min } 50 \mathrm{~s} \\
\text { Texturas de } \\
\text { alta } \\
\text { qualidade. }\end{array}$ \\
\hline Alta & $\begin{array}{l}32 \text { min } 53 \mathrm{~s} \\
\text { Alinhamento parcial com } \\
\text { nuvem bem definida. } \\
367.416 \text { pontos }\end{array}$ & $\begin{array}{l}14 \mathrm{~h} 24 \text { min } 12 \mathrm{~s} \\
\text { Nuvem rugosa com pontos } \\
\text { irregulares. } \\
122.954 .350 \text { pontos }\end{array}$ & Memória insuficiente & $\begin{array}{l}\text { Não } \\
\text { realizado }\end{array}$ \\
\hline Máxima & $\begin{array}{l}36 \text { min } 15 \mathrm{~s} \\
\text { Alinhamento parcial com } \\
\text { nuvem bem definida. } \\
334.322 \text { pontos }\end{array}$ & $\begin{array}{l}\text { Tempo de processamento } \\
\text { muito grande para formação } \\
\text { da dense cloud, superando } \\
\text { dias. }\end{array}$ & Não realizado & $\begin{array}{l}\text { Não } \\
\text { realizado }\end{array}$ \\
\hline
\end{tabular}

Fonte: autores, 2019 
Tabela 2: Comparação entre as potencialidades do Agisoft Metashape na workstation.

\begin{tabular}{|c|c|c|c|c|}
\hline $\begin{array}{l}\text { Qualidade/ } \\
\text { etapa de } \\
\text { processo }\end{array}$ & $\begin{array}{l}\text { Alinhamento (sparse } \\
\text { cloud) }\end{array}$ & Dense Cloud & Mesh & Textura \\
\hline Mínima & $\begin{array}{l}1 \text { min } 11 \mathrm{~s} \\
\text { Alinhamento } \\
\text { extremamente impreciso } \\
\text { com pontos espalhados, } \\
\text { não criando um desenho } \\
\text { claro do modelo. } \\
2.368 \text { pontos }\end{array}$ & Não realizado & Não realizado & Não realizado \\
\hline Baixa & $\begin{array}{l}2 \text { min } 31 \mathrm{~s} \\
\text { Formação do desenho } \\
\text { do modelo, porém } \\
\text { bastante impreciso com } \\
\text { pontos espalhados. } \\
82.655 \text { pontos }\end{array}$ & $\begin{array}{l}14 \text { min } 6 \mathrm{~s} \\
\text { Nuvem rugosa traz o } \\
\text { desenho do modelo, } \\
\text { mas apresenta } \\
\text { irregularidades. } \\
7.373 .265 \text { pontos }\end{array}$ & $\begin{array}{l}2 \text { min } 46 \text { s } \\
\text { Modelo precário } \\
\text { razoavelmente } \\
\text { definido, porém com } \\
\text { muita rugosidade e } \\
\text { certas irregularidades. } \\
163.849 \text { faces } \\
85.815 \text { vértices }\end{array}$ & $\begin{array}{l}4 \text { min } 18 \mathrm{~s} \\
\text { Textura bastante fiel e } \\
\text { de boa qualidade, } \\
\text { porém com } \\
\text { imprecisões e } \\
\text { rugosidades. }\end{array}$ \\
\hline Média & $\begin{array}{l}4 \text { min } 36 \mathrm{~s} \\
\text { Alinhamento melhor } \\
\text { definido formando mais } \\
\text { detalhadamente o } \\
\text { desenho do modelo. } \\
292.961 \text { pontos }\end{array}$ & $\begin{array}{l}1 \mathrm{~h} 42 \text { min } 4 \mathrm{~s} \\
\text { Nuvem mais densa } \\
\text { com maior } \\
\text { detalhamento, } \\
\text { apresentando ainda } \\
\text { irregularidades. } \\
28.626 .632 \text { pontos }\end{array}$ & $\begin{array}{l}11 \text { min } 33 \mathrm{~s} \\
\text { Modelo melhorado com } \\
\text { rugosidades evidentes } \\
\text { em algumas áreas e } \\
\text { com poucas } \\
\text { irregularidades. } \\
1.908 .437 \text { faces } \\
968.228 \text { vértices }\end{array}$ & $\begin{array}{l}8 \text { min } 19 \mathrm{~s} \\
\text { Uma boa parte das } \\
\text { rugosidades é } \\
\text { resolvida, o que } \\
\text { resulta em um modelo } \\
\text { de boa qualidade. }\end{array}$ \\
\hline Alta & $\begin{array}{l}9 \text { min } 43 \mathrm{~s} \\
\text { Desenho do modelo } \\
\text { bastante denso e } \\
\text { detalhado. } \\
367.339 \text { pontos }\end{array}$ & $\begin{array}{l}15 \text { h } 55 \text { min } 15 \mathrm{~s} \\
\text { Nuvem bastante } \\
\text { densa com grande } \\
\text { detalhamento e } \\
\text { poucos pontos } \\
\text { irregulares. } \\
119.892 .961 \text { pontos }\end{array}$ & $\begin{array}{l}57 \text { min } 54 \text { s } \\
\text { Modelo de alta } \\
\text { qualidade } \\
\text { apresentando poucas } \\
\text { áreas levemente } \\
\text { rugosas. } \\
23.978 .571 \text { faces } \\
12.059 .328 \text { vértices }\end{array}$ & $\begin{array}{l}19 \text { min } 20 \mathrm{~s} \\
\text { Modelo de alta } \\
\text { qualidade com poucas } \\
\text { irregularidades. }\end{array}$ \\
\hline Máxima & $\begin{array}{l}10 \text { min } 6 \mathrm{~s} \\
\text { Desenho do modelo } \\
\text { bastante denso e } \\
\text { detalhado. } \\
343.947 \text { pontos }\end{array}$ & $\begin{array}{l}10 \text { h } 37 \text { min } 30 \mathrm{~s} \\
\text { Nuvem bastante } \\
\text { densa com grande } \\
\text { detalhamento. } \\
577.914 .008 \text { pontos }\end{array}$ & Memória insuficiente & Não realizado \\
\hline
\end{tabular}

Fonte: autores, 2019.

Por meio dos resultados pode-se inferir que o processamento na workstation é muito mais veloz, chegando a $1 / 4$ do tempo de processamento dos dados no notebook.

$\mathrm{Na}$ qualidade mínima, tanto no notebook como na workstation, foi realizado o alinhamento das imagens sem sucesso. No primeiro caso, o programa não reconheceu o posicionamento das imagens. Na workstation, obteve-se uma nuvem de pontos muito difusa.

Nas qualidades baixa e média, ambos os hardwares conseguiram concluir o processo, gerando-se um mesh texturizado.
Nas qualidades alta e máxima, algumas etapas não foram realizadas por deficiência do hardware, isto é, os produtos gerados nas etapas exigem memória muito alta para o seu processamento. Verificou-se que na workstation, com mais memória, a qualidade alta foi concluída enquanto no notebook houve falha nesse processo.

No Agisoft Metashape a nuvem de pontos (sparse e dense cloud) pode ser usada para auxiliar o redesenho numa aplicação BIM ou mesmo como base de modelagem CAD. Tanto a nuvem de pontos quanto o mesh podem ser usados na documentação de patrimônios. 


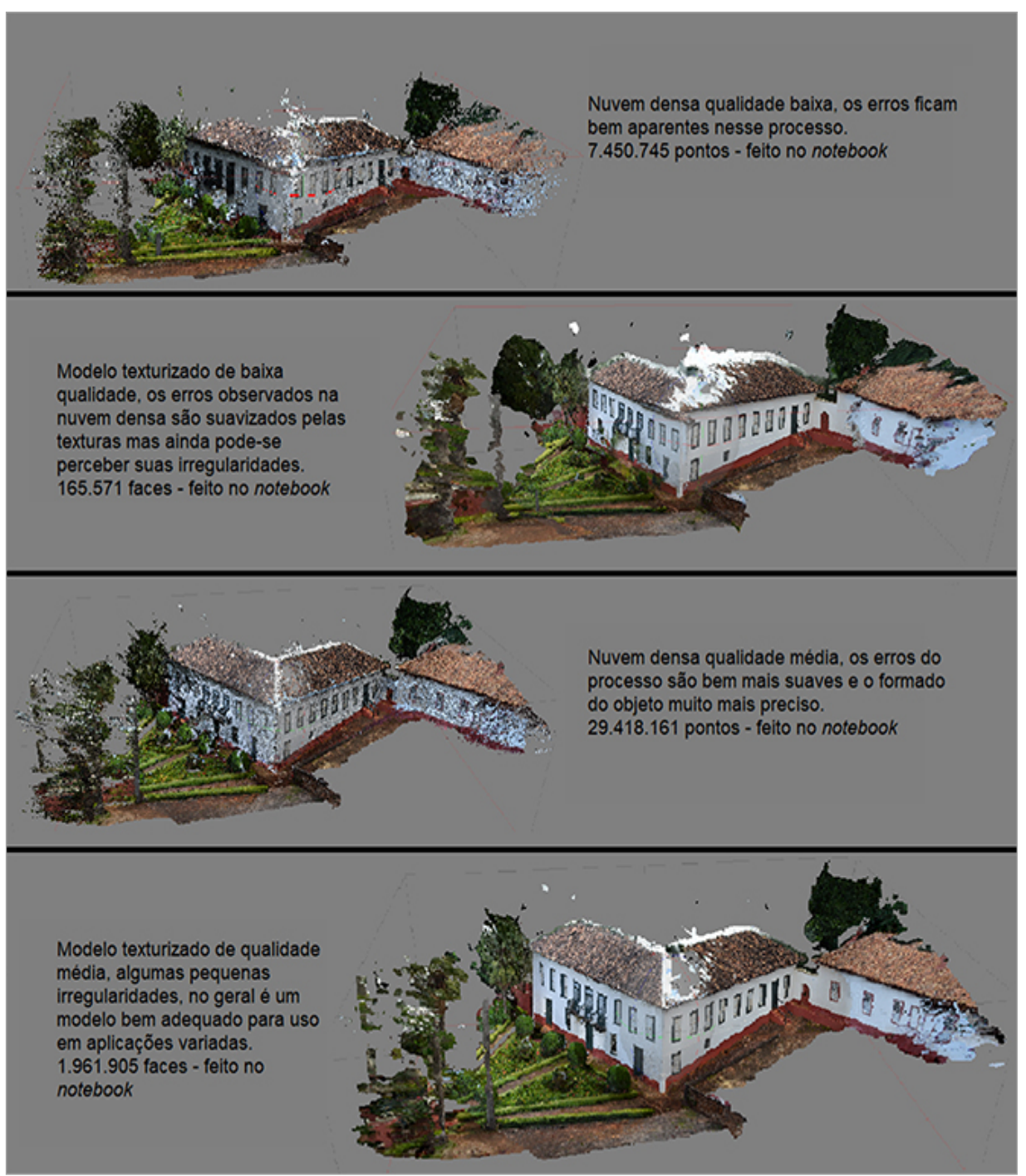

Figura 2: Processamento da fotogrametria no notebook. Fonte: autores, 2019.

As Figuras 1 e 2 ilustram os produtos do processamento de dados (das fotos) pelo Agisoft Metahape. As diferenças dos resultados nos dois hardwares, notebook e workstation, não apresentaram grandes discrepâncias entre si quanto ao número de pontos da nuvem e nem quanto ao número de faces gerado.
A diferença entre os dois testes ocorreu quanto à qualidade dos modelos, na workstation, percebeu-se uma maior grau visível dos detalhes da Fazenda. 


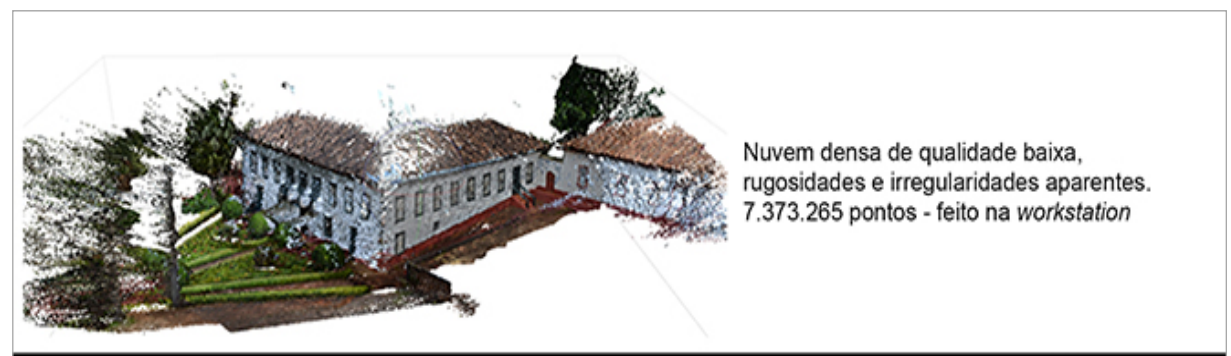

Modelo texturizado de baixa qualidade, os erros encontrados na nuvem densa são suavizados pelas texturas mas ainda há irregularidades no resultado final. 163.849 faces - feito na workstation
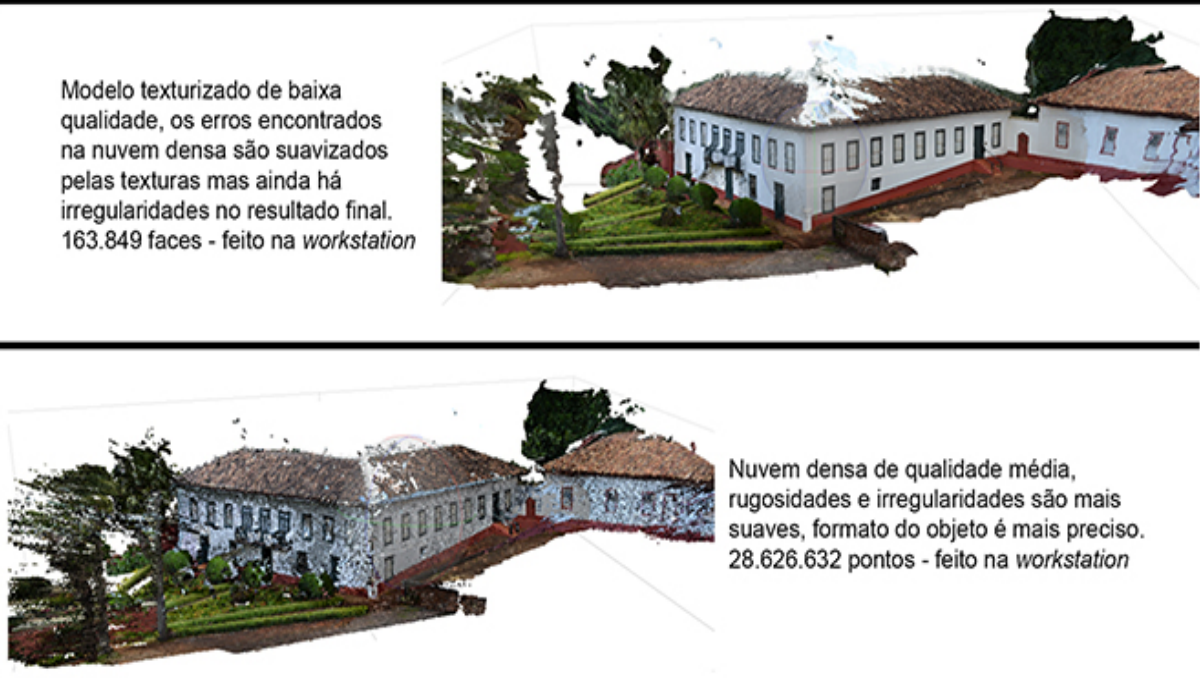

Nuvem densa de qualidade média, rugosidades e irregularidades são mais suaves, formato do objeto é mais preciso. 28.626.632 pontos - feito na workstation

Modelo texturizado de qualidade média, bem adequado para uso em aplicações variadas apesar de algumas pequenas irregularidades. 1.908 .437 faces - feito na workstation

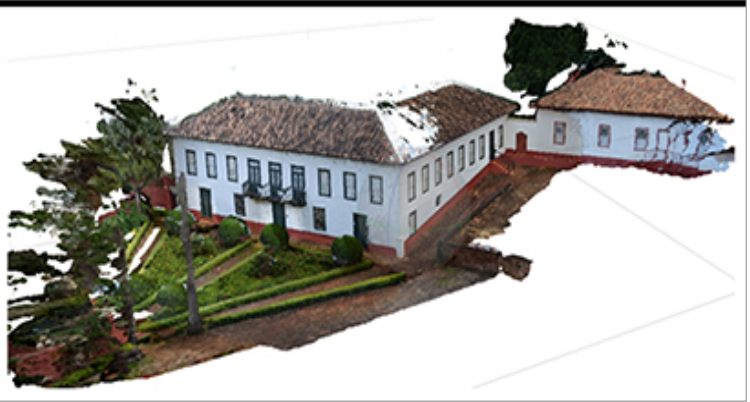

Figura 3: Processamento de fotogrametria na workstation. Fonte: autores, 2019.

\section{CONCLUSÃO}

Compreender os resultados obtidos e suas resoluções é importante para se gerenciar o tempo, recursos e a infraestrutura necessários para o desenvolvimento do projeto.

De modo geral, os resultados finais obtidos nos dois hardwares não são tão discrepantes, com exceção das qualidades mais extremas (mínima e máxima). Essas qualidades ou não produzem um resultado utilizável ou produzem resultados demasiado pesados (projetos com mais de 50GB) e inviáveis de serem processados (levando dias e superando mais de 64GB de memória RAM). Naturalmente, existe uma grande diferença entre as qualidades baixa e alta, mas a diferença entre as qualidades média e alta pode ser descartada para boa parte das aplicações.

Assim, compreender os resultados obtidos em cada opção de qualidade pode otimizar o tempo e recursos de desenvolvimento. As qualidades mais baixas podem suprir as necessidades de projetos que exijam menor precisão, por exemplo, o objeto pode ser usado como plano de fundo na renderização ou no caso de atividades com tempo limitado. Em caso de aplicações mais precisas, recomenda-se o uso das qualidades média ou alta de acordo com a precisão requerida e tempo disponível. Estas qualidades são indicadas para o acompanhamento de patologias, por exemplo.

A tecnologia da fotogrametria vem sendo amplamente utilizada e aperfeiçoada em Instituições de Pesquisa, 
como no caso da Itália, POLIMI, Firenze e Sapienza di Roma. No Brasil, alguns bons estudos já foram desenvolvidos, porém, ainda há um caminho a trilhar para superar algumas dificuldades, principalmente quanto às interfaces. Nesse sentido, este trabalho procurou apresentar alguns parâmetros que possam auxiliar outros pesquisadores. Porém, o fundamental é definir o que se pretende com os produtos da fotogrametria, para então, definir o processo de forma otimizada.

\section{AGRADECIMENTOS}

Ao IAU USP pelo suporte, ao N.ELAC pelo apoio e infraestrutura disponibilizados, à Comissão de Pesquisa do IAU USP, ao laboratório HE.SU.TECH, ao Centro de Estudos da Casa do Pinhal e à Fundação de Amparo à Pesquisa do Estado de São Paulo (FAPESP) Processo no. 18958-0 pelo apoio financeiro.

\section{REFERÊNCIAS}

Almeida, A.; Silva, G.; Vizioli, S. Photogrammetry as a tool for cultural heritage valorization: Workshop at Casa do Pinhal. HBIM 2019. São Carlos.

Amorim, A. L.; Groetelaars, N.A fotogrametria digital na documentação do patrimônio arquitetônico. In: Fórum Patrimônio: ambiente construído e patrimônio sustentável, Belo Horizonte, MG. Anais... Belo Horizonte: Vol.2, N.2, 2008, p. 92-105.
Bastian, A. V. Fotogrametria esférica: uma técnica de baixo custo para documentação arquitetônica. In Anais... SIGraDi 2016, XX Congress of the Iberoamerican Society of Digital Graphics - Buenos Aires. Ed. Blucher, 2016.

CARPO, Mario. The second digital turn: design beyond intelligence. Cambridge, MA: The MIT Press, 2017.

Fassi, F.; Fregonese, L.; Ackermann, S.; de Troia, V. Comparison between laser scanning and automated $3 d$ modelling techniques to reconstruct complex and extensive cultural heritage area. In: ISPRS Annals of Photogrammetry, 2013. Anais... Trento: 2013, p. 73-80.

Righi, T. A. F; Celani, G. Esboços na era digital: uma discussão sobre as mudanças na metodologia de projeto arquitetônico. Congresso Iberoamericano de gráfica digital SIGRADI. In Anais... Havana Cuba 2008.

Peterson, L. Laser Scans could help rebuild Notre Dame Cathedral. The Atlantic, 16 de Abril de 2019. Entrevista concedida a Alexis C. Madrigal. Disponível em <https://www.theatlantic.com/technology/archive/2019/04// aser-scans-could-help-rebuild-notre-dame-

cathedral/587230/> Acessado em: 08 de Setembro de 2019.

Shults, R. Development and implementation of innovative educational modules on architectural photogrammetry for bachelor's degree curricula in Architecture. In Proceedings The International Archives of the Photogrammetry, Remote Sensing and Spatial Information Sciences. Bergamo, 2019 